\title{
Funding Research at the State Level: Hawaii's Industry-analysis Process
}

\author{
Noel P. Kefford \\ College of Tropical Agriculture and Human Resources, University of Hawaii, Honolulu, HI 96822
}

\author{
H.C. Bittenbender \\ Department of Horticulture, University of Hawaii, Honolulu, HI 96822
}

The prominence and prestige of federal funding for research do a disservice to state-level funding. Statistics show that a major part of faculty salaries and other horticultural research expenses comes from in-state sources. However, the real funding issue before us today is not federal vs. state funding, it is the total package of funding for horticultural research. All sources: federal, state, or private; formula, grant, or contract should contribute in a complementary manner to the optimal package. We believe that the principles of our message apply to the package and all of its components, even though the specifics relate to our experience in Hawaii with state priority setting and funding.

Whatever its source, research funding places a set of responsibilities upon faculty and administrators. The responsibilities are broader than responsible spending after funding is received, because the most critical steps in constructing a funding package occur before individual investigators receive their budgets. Our thesis is that all steps of the research-funding process need objective, unbiased scientific knowledge. For knowledge of horticulture, faculty are the source. Based on experience in Hawaii, research and extension faculty have a great opportunity to use the power of this knowledge to influence the determination of research policies and funding.

All research funding has its origin in the process of setting priorities. However, your research funding is not determined by priorities set in your own laboratory, department, college, or university. Rather, these priorities relate ultimately to policies and values established outside your institution. For example, federal formula funding for agriculture is based on the policy of federal and state cooperation in priority setting and funding.

There is a sequence of steps that lead from policy and values to the funding of research (Table 1). Note that when a policy is in place, the system diagrammed in Table 1 first calls for the definition of opportunities and constraints. A policy that has permitted our college to increase its impact on Hawaii's agriculture was enacted by the legislature in 1976 . The essence of the policy states that agricultural development in Hawaii shall be based upon systematic planning and priority setting and upon coordinated implementation of priorities by the public and private sectors. A subgroup of the governor's cabinet, the Governor's Agriculture Coordinating Committee (GACC), was established in the statute to carry out the policy. However, the state did not have a process for systematic planning, priority setting, and coordinated implementation. Our college relieved that constraint. We developed a process for analyzing a commodity industry. The college could offer this process to the state only because it had the collective expertise of its faculty. Indeed, the industry-analysis process provides the vehicle for our faculty to carry out their responsibilities at each step of the sequence in Table 1.

We will summarize the process and give particular attention to the roles of faculty. In Hawaii, the process has been applied to 25 plant and animal industries plus processed foods and forest products. Some commodities have been analyzed five times over a span of 14 years.

The analysis of a commodity industry that leads to priorities must be based on a projection of the industry's economic potential. Faculty have the expertise to assist an industry to become aware of its options for the future and to choose targets toward which it can move. That is an appropriate function for horticulture faculty-the function of making wise choices among options.
Options are packages of opportunities and constraints. In the industry-analysis process, the potentials of industries are the opportunities and the constraints are the bottlenecks to the achievement of those potentials. Hawaiian crops have potentials ranging from strong to unknown, and, in each case, achievement of their potential requires the relief of bottlenecks. Hawaiian coffee, for instance, has potential to increase $100 \%$ in the next few years, provided that plantation culture and mechanical harvesting can produce a distinctive, high-quality product. The future of the export papaya industry is unknown pending the approval of a new quarantine procedure for fruit-fly disinfestation that, fortunately, appears very close. The expertise of research and extension faculty is needed to assure that projections of future industry potentials are based on sound assumptions.

The next phase of analysis begins with defining key components, for example, those for a typical horticultural industry (Table 2). Then the specialized expertise of faculty can be focused on each component. The outcome of this interdisciplinary effort is a summary of all that is known and unknown about an industry. From this summary, the specific bottlenecks to an industry reaching its potential become self-evident. The insect problems of macadamia provide an example. Insect damage to nuts recently has become a factor of quality that is critical to this upscale industry. However, the extent of damage by each candidate pest in each growing area is unknown. Also, the biology of some pests is unknown because they only recently have had a significant impact on macadamia. Therefore, one bottleneck is the lack of crop-loss assessment data. A further bottleneck is the lack of biological information necessary to develop management strategies for the pests. The actions required to relieve both bottlenecks are to initiate the needed research.

Responsibility for completing these actions was given jointly to

Table 1. Steps leading from policies and values to the funding of research and the use of research results.

\begin{tabular}{l}
\hline Policy/values \\
Opportunity to implement policy \\
Constraints to opportunity \\
Priority actions to relicve constraints \\
Funding for researchable actions \\
Constraints relieved \\
Opportunities realized \\
Policy/values justified \\
\hline
\end{tabular}

Table 2. Components of a horticultural industry.

\begin{tabular}{l} 
Land and water \\
Labor \\
Finance \\
Cultivars \\
Pests and diseases \\
Weeds \\
Culture \\
Harvesting and postharvest \\
Processing and marketing \\
Management and profitability \\
Industry organization \\
\hline
\end{tabular}


our college, the macadamia industry, and the Hawaii Dept. of Agriculture. The requirement for joint action illustrates the important point that industry analysis is comprehensive in that it draws upon the resources of all contributing agencies.

Actions to relieve bottlenecks commonly require both existing and additional faculty, technician time, and funds for equipment and operations. Such projections must be made for the period of the project. These data become critical when bottlenecks and their actions are being prioritized and cost/benefit is being assessed. In the analysis, the benefit portion of cost/benefit is an estimate of the impact of taking the action to relieve the bottleneck. Faculty can help specify the impact of adopting the anticipated results of their research. Also, their estimates of the cost of the research are the cost portion of cost/benefit.

The input of the faculty to the development of the analysis ends at this point, and the primary responsibility moves to the industry. This brings us to emphasize that we have been using the term research to include research delivery and adoption. Therefore, both research and extension faculty have essential roles in the industry analysis process.

The transition from faculty to industry responsibility culminates in a 1-day meeting during which a faculty-generated, draft document is transformed into the industry's own analysis and action plan in which $\approx 20$ bottlenecks are listed in priority order. As all components of an industry from basic resources to the retailing of products must be considered, representation from all components must be at this meeting. Amazingly, consensus can be reached by the disparate group in just a few hours. The secret is that our faculty are able to provide well-organized information as the basis for discussions and priority setting by the industry's representatives. We know the process works because we have conducted dozens of these industry meetings.

As the next step, the industry presents its analysis and action plan to the GACC. This committee is responsible for assuring that the industry's priorities and actions are in accord with overall agriculture development plans of the state. The analysis and action plan that the committee accepts establishes state policy and practice for the industry for 3 years, which is the period between analyses. Also, the committee receives funds from the Hawaii legislature specifically to fund the top priority actions. To carry out many of these actions requires research, extension, or training, all of which in- volve faculty. The faculty must develop the scientific project proposal that will achieve an action. An example is an action to improve the disease resistance of Anthurizum for which a researcher submits a project based on the techniques of biotechnology. This project is the basis for a contract with the GACC. The contract requires feedback to industry.

What are the benefits of all of this effort? The benefits to the college's Dept. of Horticulture have been: 1) the GACC has become the largest component of nonsalary funding, 2) the faculty interact systematically with representatives of industry and other disciplines, and 3) research results are integrated into packages ready for realworld testing. Industry and the state benefit because 1) systematic planning has replaced pressure groups in setting state priorities, 2) state resources support research with the best chance to assist economic development and improve the environment, 3) state, federal, and private resources are used to achieve commonly determined objectives, and 4) all components of an industry are brought together to make choices on the priorities for an industry to reach its potential.

You must be asking how the industry analysis process would apply in your department, university, and state. The fact is that the elements of the process are present in all states. These elements must be used systematically and comprehensively so that they can relate to policy, priority setting, and research funding. The commodity industry analysis process has provided the vehicle for Hawaii to bring order to the state's policy/priority setting/support sequence for agriculture. We believe that you can use the approach that led Hawaii to the industry-analysis process that matches both your state's needs and its decision-making context.

We also have direct experience with the application of the basic process of commodity industry analysis to circumstances very different from those in Hawaii. We used it to analyze the wheat industry of Jordan. The Hawaii process had to be adapted to conditions in Jordan and the same would apply to its use in any other country or state.

The commodity industry analysis process has been shown to increase the in-state contribution to the total research package in an open, systematic way. The approach could do the same for other components of the total U.S. research funding for mission-oriented and basic horticultural research. 